\title{
Análisis y comparación de la Competencia Transversal Análisis y Resolución de Problemas en asignaturas de Grado
}

Silvia Alvarez Blanco ${ }^{\mathrm{a}}$, Ignacio Bosch Roig ${ }^{\mathrm{b}}$, Cristina Jordan Lluch', Jaime Lloret ${ }^{\mathrm{b}}$, José Antonio Mendoza ${ }^{a}$, Lucia Romero Perez ${ }^{\mathrm{d}}$, Esther Sanabria-Codesal ${ }^{\mathrm{c}}$ y Maria Cinta Vincent-Vela ${ }^{\mathrm{a}}$

a'Departamento de Ingeniería Química y Nuclear (sialvare@iqn.upv.es, mavinve@iqn.upv.es, jamendoz@iqn.upv.es), b ${ }^{\mathrm{b}}$ Departamento de Comunicaciones (igbosroi@dcom.upv.es, jlloret@dcom.upv.es), 'Departamento de Matemática Aplicada (cjordan@mat.upv.es, esanabri@mat.upv.es) y ${ }^{\mathrm{d}}$ Departamento de Ingeniería Electrónica (lurope@eln.upv.es)

\begin{abstract}
The last syllabus of the grades of the Polytechnic University of Valencia (UPV) has changed the perspective of the universitary teaching and has highlighted the importance of the specific and the transversal competences. Therefore, there is a need for grading the transversal competences throughout the degrees and masters of the UPV. Moreover, these transversal competences must be assessed in several subjects. Due to the lack of experience in evaluating these skills, UPV has elaborated scoring rubrics to help professors to assess them. In this work, we review the assessment of the transversal competence "Problem identification and solving" of four subjects of different degrees and courses. In two subjects, special activities have been performed for the evaluation of this competence and scoring rubrics have been used. In the other two subjects, the mark of this skill has been obtained from other marks of the subject. Moreover, we have compared the mark of this skill with the final mark of the subject. Our results show that there is a high correlation between the two marks in the four subjects.
\end{abstract}

Keywords: Transversal skills, Problem Solving, Grade.

\section{Resumen}

En los últimos planes de estudios de los grados de la Universidad Politécnica de Valencia (UPV) ha cambiado el enfoque de la docencia y se ha resaltado el papel de las competencias, tanto específicas como transversales. Actualmente es necesario evaluar competencias transversales en todos los grados y másteres de la UPV y estas deben ser evaluadas en distintas asignaturas. Ante la falta de experiencia en su evaluación, la UPV ha elaborado rúbricas para ayudar al profesorado en dicha tarea. En este artículo revisamos la evaluación de la competencia transversal "Análisis y resolución de problemas" en cuatro asignaturas de distintos cursos y grados. En dos de estas asignaturas se han realizado actividades especiales para la evaluación de esta competencia y se han utilizado rúbricas para ello. En las otras dos asignaturas, se ha optado por utilizar las calificaciones de los actos de evaluación más relacionados con ella. Además, se ha comparado la calificación de esta competencia con la nota de la asignatura y los resultados 
muestran que para las cuatro asignaturas existe una alta correlación entre ambas.

Palabras clave: Competencias transversales, Resolución de problemas, Grado.

\section{Introducción}

La última modificación de planes de estudio ha traído consigo, entre otras cosas, un importante cambio en la manera de evaluar a nuestros alumnos (Sendra et al., 2009). Hemos pasado de centrarnos en un listado de contenidos que nuestros estudiantes debían conocer para superar con éxito sus asignaturas, a una orientación completamente diferente basada en resultados de aprendizaje y el desarrollo de competencias (Lloret et al., 2006).

Según el Real Decreto 1125/2003 (2003): "Los créditos ECTS representan el volumen de trabajo del estudiante para conseguir los objetivos del programa de estudios, objetivos que deben ser especificados preferiblemente en términos de resultados del aprendizaje y de competencias que han de ser adquiridas". Por tanto, para acreditar los títulos de Grado ha sido necesario definir las competencias asociadas y asignarlas a las diferentes asignaturas.

Existen dos tipos de competencias: transversales y específicas. Las competencias específicas son las relacionadas directamente con la ocupación, por tanto están asociadas con las asignaturas que se imparten en cada Grado. Las competencias transversales, siguiendo lo propuesto por González y Wagenaar (2003), se definen como aquellas habilidades relacionadas con el desarrollo personal, que no dependen de un ámbito temático o disciplinario específico sino que aparecen en todos los dominios de la actuación profesional y académica. Por tanto, este término indica capacidades que no son específicas de una profesión en concreto y son aplicables a tareas y contextos distintos. Luego estas competencias pueden estar distribuidas en varias asignaturas de ámbitos muy diferentes (Sanabria-Codesal et al., 2014).

La Universitat Politècnica de València (UPV) ha considerado que las competencias transversales que sus egresados deben tener son las siguientes (UPV, 2016):

CT UPV1: Comprensión e integración

CT UPV2: Aplicación y pensamiento práctico

CT UPV3: Análisis y resolución de problemas

CT UPV4: Innovación, creatividad y emprendimiento

CT UPV5: Diseño y proyecto

CT UPV6: Trabajo en equipo y liderazgo

CT UPV7: Responsabilidad ética, medioambiental y profesional

CT UPV8: Comunicación efectiva

CT UPV9: Pensamiento critico

CT UPV10: Conocimiento de problemas contemporáneos

CT UPV11: Aprendizaje permanente

CT UPV12: Planificación y gestión de tiempo

(cc) EY-NC-ND 2016, Universitat Politècnica de València

Congreso IN-RED (2016) 
La correcta adquisición de ellas se establece en tres niveles de dominio: el primero corresponde a asignaturas de 1 y 2 de grado, el segundo a asignaturas de 3 y 4 y el tercero con asignaturas de Máster.

El resto del artículo está estructurado como sigue a continuación. En la sección 2 se muestran los objetivos que se persiguen en este trabajo. En la sección 3 se explica la competencia transversal “Análisis y resolución de problemas” y en la sección 4 se detalla su evaluación en las diferentes asignaturas. La sección 5 muestra los resultados obtenidos y finalmente, la sección 6 incluye las conclusiones y posibles trabajos futuros.

\section{Objetivos}

En este trabajo nos centraremos en la evaluación de la competencia transversal CT3: “Análisis y resolución de problemas” en diferentes asignaturas, niveles y grados de la UPV. Las asignaturas donde se ha llevado a cabo esta experiencia se muestran en la Tabla 1.

Tabla 1. Asignaturas en las que se ha trabajado la competencia transversal CT3

\begin{tabular}{|c|c|c|c|}
\hline Asignatura & Grado & $\begin{array}{c}\text { Curso / Nivel } \\
\text { competencia }\end{array}$ & Cuatrimestre \\
\hline $\begin{array}{c}\text { Matemática } \\
\text { Discreta }\end{array}$ & Grado en Informática & $1^{\circ}$ Grado / Nivel I & $\mathrm{A}$ \\
\hline Señales y Sistemas & $\begin{array}{c}\text { Grado en Ingeniería de } \\
\text { Tecnologías y Servicios de } \\
\text { Telecomunicación }\end{array}$ & $2^{\circ}$ Grado / Nivel I & $\mathrm{A}$ \\
\hline Matemáticas II & Grado en Ingeniería Mecánica & $2^{\circ}$ Grado / Nivel I & $\mathrm{A}$ \\
\hline $\begin{array}{c}\text { Reactores } \\
\text { químicos }\end{array}$ & Ingeniería Química & $3^{\circ}$ Grado / Nivel II & $\mathrm{A}$ \\
\hline
\end{tabular}

El objetivo de este trabajo es analizar los diferentes enfoques a la hora de evaluar esta competencia transversarl en las asignaturas.

\section{Análisis y resolución de problemas}

Siguiendo la clasificación establecida en el proyecto Tuning la competencia transversal “Análisis y resolución de problemas" se encuentra entre las denominadas competencias instrumentales. Los estudiantes necesitan ser capaces de aplicar lo que han aprendido en situaciones nuevas y por tanto la adquisición de esta competencia proporciona una sólida base para el aprendizaje futuro de los egresados y para su participación efectiva en la sociedad (OECD, 2010). Se puede definir como "la capacidad individual para participar en el procesamiento cognitivo para comprender y resolver situaciones en las que un método de solución no es inmediatamente evidente. Esto incluye la voluntad para participar en tales situaciones con el fin de conseguir el propio potencial como ciudadano reflexivo y consecuente". 
Para adquirir esta la competencia, la Universitat Politècnica de València recomienda diversas actividades formativas para trabajar con los alumnos (UPV ICE, 2016): actividades grupales, estudio de casos, exposiciones orales, lecturas, prácticas de laboratorio, preguntas, problemas, proyectos, seminarios, etc. Por otra parte, la evaluación puede realizarse también por numerosos sistemas, por lo que se puede decir que hay una gran variedad de formas de trabajar y evaluar la competencia, siendo de máximo interés los estudios comparativos con el fin de determinar de qué forma los estudiantes pueden adquirir un mejor dominio de esta competencia.

En este sentido, Vicent et al. (2006) relacionaron la adquisición de esta y otras competencias transversales en el marco de herramientas de aprendizaje en la red, es decir, en el denominado “e-learning”, mientras que Giordano y Maiorana (2014) desarrollaron su estudio en el marco del aprendizaje de los lenguajes de programación. Sánchez-Barba et al. (2013) emplearon con éxito problemas con dificultad creciente (desde problemas totalmente definidos a problemas complejos) para que los alumnos de la asignatura de química trabajaran y adquieran la competencia de resolución de problemas.

Gleason et al. (2013) evaluaron mediante rúbricas la adquisición de las competencias “pensamiento crítico” y “Análisis y resolución de problemas” por parte de alumnos de doctorado en farmacia. En particular, para la resolución de problemas emplearon una rúbrica de 6 criterios, asignando una calificación entre 1 y 4 para cada criterio. Los resultados sirvieron para mejorar el plan de estudios con vistas a una mayor adquisición de la competencia por parte de los estudiantes.

Cabe comentar que algunos autores distinguen entre "resolución de problemas" y “resolución de problemas complejos”, recomendando para este último fin el trabajo en grupo más que actividades de carácter individual. Tal es el caso de Hung (2013).

\section{Evaluación de la competencia en las asignaturas}

En esta sección se presentan todas las asignaturas donde se ha trabajado la competencia “Análisis y resolución de problemas” y se explica la forma de evaluación de esta competencia en cada una de ellas.

\subsection{Matemática Discreta}

La competencia “Análisis y resolución de problemas” es evaluada en el primer curso del grado en Ingeniería Informática que se imparte en la ETSINF de la UPV en la asignatura Matemática Discreta (MAD) correspondiente al primer cuatrimestre. Dadas sus características, esta asignatura es idónea para constituir ello.

Esta asignatura tiene asignados 4,5 créditos en aula y 1,5 en laboratorio informático. El temario impartido contiene los temas: Lógica, Conjuntos, Relaciones binarias, Divisibilidad, Álgebras de Boole y Combinatoria, dedicando las sesiones de laboratorio al estudio introductorio de la Teoría de Grafos.

(cc) EY-NC-ND 2016, Universitat Politècnica de València 
Uno de los objetivos de la asignatura MAD, a cuya consecución se dedica una gran parte del tiempo, coincide con el nombre de esta competencia transversal, Análisis y resolución de problemas, lo que por otra parte resulta habitual en las asignaturas de Matemáticas. A lo largo del curso se resuelven numerosos problemas y estudio de casos, y se proponen ejercicios para resolver tanto dentro como fuera del aula. Este año la evaluación se ha llevado a cabo a partir de la nota obtenida en el examen correspondiente a las sesiones de aula, de manera proporcional a la calificación obtenida en este. Está previsto para el próximo curso obtener la valoración de la competencia a partir de preguntas concretas del examen y/o problemas planteados, resueltos y recogidos en el aula.

\subsection{Señales y Sistemas}

La asignatura Señales y Sistemas es una de las asignaturas de formación básica de la materia básica de Telecomunicación, del segundo curso (cuatrimestre A) del grado en Ingeniería de Tecnologías y Servicios de Telecomunicación. Tiene asignados 4,5 créditos de teoría en el aula y 1,5 créditos de prácticas.

En ella se presenta la teoría se señales y sistemas de tiempo continuo y discreto, necesaria para el modelado de los elementos básicos que aparecen en telecomunicaciones. Los conceptos presentados son fundamentales en el desarrollo de gran parte de las asignaturas que posteriormente se cursan en la titulación, en especial de aquellas relacionadas con el ámbito del análisis y tratamiento de señales. Desde esa perspectiva podemos considerar la asignatura como una transición entre las herramientas puramente matemáticas y su orientación hacia el ámbito específico de las telecomunicaciones. En definitiva los conceptos presentados constituyen un elemento esencial en la formación básica de la titulación.

En la asignatura se trabajan diversas competencias específicas de la materia básica de telecomunicación, siendo punto de control de dos de ellas. Así como dos competencias transversales de las que también es punto de control. Concretamente la CT2: Aplicación y pensamiento práctico, trabajada en las prácticas de la asignatura y la CT3: Análisis y resolución de problemas que nos ocupa y trabajada en los problemas de clase y en los diversos problemas de los tres parciales que evalúan la parte teórica de la asignatura.

Por lo tanto se han aprovechado los tres parciales de la asignatura, para incorporar de forma integrada la formación y evaluación de la competencia transversal CT3. Los contenidos sobre los que se va a trabajar son los propios de la asignatura que por otro lado ya se evaluaban de forma habitual con problemas. Por lo que se han incorporado ciertas preguntas a algunos apartados de los problemas que destacan la importancia de incorporar, en la resolución del ejercicio, los resultados de aprendizaje de la competencia transversal.

Para la evaluación de "Análisis y resolución de problemas" se han seguido los siguientes resultados de aprendizaje que se basan en una rúbrica elaborada a tal efecto. Cada uno de los indicadores se han evaluado con la calificación D, C, B y A, siendo D la calificación de no alcanzado y A la calificación de excelente.

(cc) EY-Nc-ND 2016, Universitat Politècnica de València

Congreso In-Red (2016) 
Resultado de aprendizaje 1 (RA1): Determinar si algún dato es innecesario, redundante (se puede deducir de otros) o contradictorio. Siendo una D si no se determina correctamente los datos innecesarios y una A si los determina correctamente.

Resultado de aprendizaje 2 (RA2): Ser capaz de realizar un esquema o gráfico que ayude a la comprensión del problema. Siendo una D si lo realiza y una A si lo realiza y es correcto.

Resultado de Aprendizaje 3 (RA3): Ser capaz de realizar una verificación rápida del resultado para detectar posibles errores durante el desarrollo. Siendo una D si no comprueba los resultados ni el procedimiento y una A si los comprueban y los contrasta

Resultado de Aprendizaje 4 (RA4): Ser capaz de determinar si un problema se puede resolver de varios modos y valorar cada una de las alternativas desde distintos puntos de vista. Siendo una D si no presenta alternativas habiendo elegido una solución poco eficiente y una A si presenta múltiples alternativas.

Por lo tanto, para la valoración final de la competencia se han tenido en cuenta los siguientes aspectos en orden de importancia creciente: destacar los datos innecesarios (RA1), la realización de esquemas ilustrativos (RA2), comprobar y contrastar el resultado obtenido (RA3) y aportar diferentes opciones de resolución (RA4).

Fijando la nota final de la compentencia transversal CT3 con el siguiente criterio:

Se ha valorado a cada alumno por separado, utilizando las 4 valoraciones de la CT3 disponibles con notas de evaluación del tercer y último parcial correspondientes a cada uno de los resultados de aprendizaje a trabajar. Dejando la valoración de la CT3 del primer y segundo parcial para casos dudosos. Ya que se ha entendido que los dos primeros parciales eran una primera aproximación más formativa, y en el último ya se han utilizado los datos para realizar la propia evaluación de la competencia.

De entre los resultados de aprendizaje se ha valorado con un mayor peso los dos últimos (RA3 y RA4), al entender que la verificación de la solución y la proposición de diferentes soluciones posibles son de un nivel mayor que la determinación de datos innecesarios y la realización de esquemas o dibujos. Es decir que se han tenido en cuenta básicamente las valoraciones de los RA3 y RA4 y se ha subido o bajado un nivel en función de la evolución de los otros dos RA1 y RA2. En el caso de que no hubiese valoraciones de algún problema, se ha considerado el valor de los otros RA a la baja.

\subsection{Matemáticas II}

Esta asignatura forma parte del plan de estudios del Grado en Ingeniería Electrónica y Automática de la Escuela Técnica Superior de Ingeniería del Diseño (ETSID) y se imparte en el primer cuatrimestre del segundo curso. Tiene asignados 4 créditos de teoría y práctica de aula y 2 créditos de prácticas de laboratorio. Está distribuida en 3 grupos con un total de 139 alumnos.

La materia de estudio de la asignatura Matemática II es ecuaciones diferenciales, transformada de Laplace y sus aplicaciones, conceptos que sirven como herramientas 
básicas para otras asignaturas del grado. Por ello la ETSID nos ha elegido como punto de control de primer nivel de la competencia transversal "Análisis y resolución de problemas”. La evaluación que se llevado a cabo en la asignatura de esta competencia está totalmente integrada en la evaluación de la materia, puesto que desde nuestro punto de vista es la única forma de desarrollarla adecuadamente y sin excesiva sobrecarga de trabajo ni para el profesorado, ni para el alumnado.

La competencia se ha trabajado mediante ejercicios realizados en clase, que son útiles para valorar otras competencias específicas de la asignatura como: "Resolver problemas matemáticos que puedan plantearse en la ingeniería” y/o "Aprendizaje permanente: aprender nuevos métodos y teorías que se adapten a nuevas situaciones”. Al finalizar cada tema los alumnos realizaban ejercicios individuales y/o en grupo. La competencia transversal que estamos analizando se desarrolla prestando una especial atención al correcto análisis y planteamiento de estos problemas.

La evaluación ha sido realizada a través de la rúbrica facilitada por la UPV para esta competencia, considerando cuatro indicadores principales:

- Define el problema describiendo de manera clara los aspectos más importantes

- Utiliza fuentes de información indicadas y selecciona los datos correctos

- Emplea un método aprendido para la resolución del problema propuesto

- Analiza la coherencia de las soluciones obtenidas

En la rúbrica, que los alumnos conocen desde el principio de curso, aparece claramente explicado el grado de cumplimiento que deben alcanzar en cada ítem para obtener las distintas puntuaciones consideradas (A excelente, B bien, C en desarrollo y D no alcanzada).

Los ejercicios de clase sirven como preparación para evaluar finalmente la competencia en los realizados en los parciales de la asignatura los cuales representan un $60 \%$ de la nota final. Por tanto, la nota obtenida en la competencia viene dada directamente de manera proporcional a este porcentaje de la nota final, donde se contemplan además, con el restante $40 \%$, los aspectos teóricos y las prácticas de laboratorio de la asignatura.

\subsection{Reactores Químicos}

La asignatura de Reactores Químicos es una asignatura obligatoria que consta de 4.5 créditos ( 2.5 créditos de teoría y 2 créditos de prácticas) y se imparte en tercer curso del Grado en Ingeniería Química. La competencia “Análisis y resolución de problemas” se evalúa mediante la realización de 4 problemas ( 2 de ellos en el primer parcial y otros 2 en el segundo parcial). Estos 4 problemas representan el $70 \%$ de la nota final de la asignatura. La nota de la competencia corresponde a la obtenida por los alumnos en estos problemas.

A la vez que se evalúa esta competencia transversal se están evaluando tres resultados de aprendizaje que se detallamos a continuación junto con el tipo de problema planteado que los evalúa:

(cc) BY-NC-ND 2016, Universitat Politècnica de València

Congreso In-Red (2016) 


\section{PROYECTOS DE INGENIERÍA}

1) La capacidad de aplicar sus conocimientos para plantear y llevar a cabo proyectos que cumplan unos requisitos previamente especificados.

Problema de examen de diseño de un sistema de reactores para alcanzar una determinada producción. Selección de la configuración y tipo de reactor adecuado que ncluye optimización económica.

2) Comprensión de los diferentes métodos y la capacidad para aplicarlos.

Problema de examen de cálculo de la máxima concentración de producto deseado en un reactor. Es necesario usar métodos numéricos para resolver las ecuaciones diferenciales.

Problema de examen de aplicación de modelos de flujo no ideal para calcular la conversión en un reactor real. Comparación del resultado de la evaluación mediante distintos modelos.

Problema de examen de evaluación de la desviación en el comportamiento de un reactor real frente a un reactor ideal. Uso de varios modelos diferentes.

\section{APLICACIÓN PRÁCTICA DE LA INGENIERÍA}

3) La comprensión de métodos y técnicas aplicables y sus limitaciones.

Problema de examen de diseño de un sistema de reactores mezcla perfecta en serie. Dimensionamiento del reactor que consiste tanto en calcular el volumen como en el cálculo de la composición de la corriente de salida.

Problema de examen de diseño de un reactor discontinuo. Cálculo del tiempo necesario para alcanzar una determinada conversión. Análisis de estabilidad en un reactor mezcla perfecta adiabático.

\section{Análisis de la competencia transversal Análisis y resolución de problemas en diferentes asignaturas}

En esta sección se analizan los resultados obtenidos por los alumnos en la evaluación de la competencia transversal considerada en las asignaturas introducidas previamente.

Las siguientes tablas y figuras muestran los resultados obtenidos tras evaluar la competencia en las diferentes asignaturas. Las segundas columnas de las tablas, denominadas Media, hacen referencia a la media de las notas finales en la asignatura obtenidas por los alumnos con la misma calificación en la competencia. De forma análoga el resto de columnas de dicha tabla son relativas a las dichas notas finales.

En particular la Tabla 2 y Figura 1 se recogen los datos relativos a la asignatura de Matemática Discreta que corresponde al nivel I de dominio de la competencia.

En ella se puede apreciar que existe una alta correlación entre la nota final y la valoración de la competencia transversal, lo que es esperable ya que se ha obtenido de forma proporcional a un $70 \%$ de la nota final de la asignatura.

(cc) EY-NC-ND 2016, Universitat Politècnica de València 
Silvia Alvarez Blanco, Ignacio Bosch Roig, Cristina Jordan Lluch, Jaime Lloret, José Antonio Mendoza, Lucia Romero Perez, Esther Sanabria-Codesal,Maria Cinta Vincent-Vela

Tabla 2. Datos referentes a la asignatura Matemática Discreta

\begin{tabular}{|c|c|c|c|c|c|c|}
\hline Matemática Discreta & Media & Desviación típica & Max caja & Min caja & Max & Min \\
\hline D & 2,72 & 0,84 & 3,55 & 1,88 & 6,1 & 0,8 \\
\hline C & 4,17 & 0,24 & 4,42 & 3,93 & 5 & 4 \\
\hline B & 5,93 & 0,76 & 6,70 & 5,17 & 8 & 5 \\
\hline A & 8,42 & 0,68 & 9,10 & 7,75 & 10 & 7,6 \\
\hline
\end{tabular}

\section{Matemática Discreta: CT03 vs Nota Final}

$\square \mathrm{D} \square \mathrm{C} \square \mathrm{B} \square \mathrm{A}$

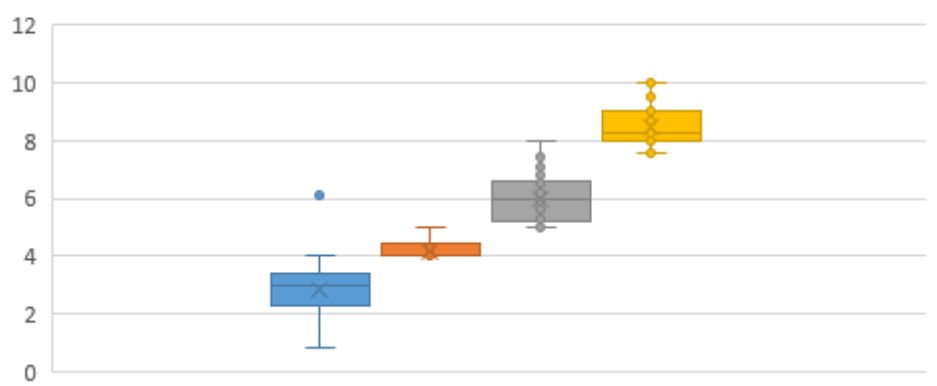

Fig. 1 Evaluación de la competencia en la asignatura Matemática Discreta

En la Tabla 3 y Figura 2 se muestran los resultados en la asignatura Señales y Sistemas.

Se puede observar que también existe una alta correlación entre la nota de la asignatura y de la competencia, a pesar de que no se ha utilizado ninguna fórmula directa para obtener la nota de esta.

Tabla 3. Datos referentes a la asignatura Señales y Sistemas

\begin{tabular}{|c|c|c|c|c|c|c|}
\hline Señales y Sistemas & Media & Desviación típica & Max caja & Min caja & Max & Min \\
\hline D & 2,52 & 0,55 & 3,07 & 1,97 & 3,18 & 1,78 \\
\hline C & 4,39 & 1,24 & 5,63 & 3,15 & 8,51 & 2,22 \\
\hline B & 5,91 & 1,21 & 7,12 & 4,70 & 6,4 & 2,22 \\
\hline A & 7,45 & 1,21 & 8,66 & 6,23 & 9,64 & 5 \\
\hline
\end{tabular}

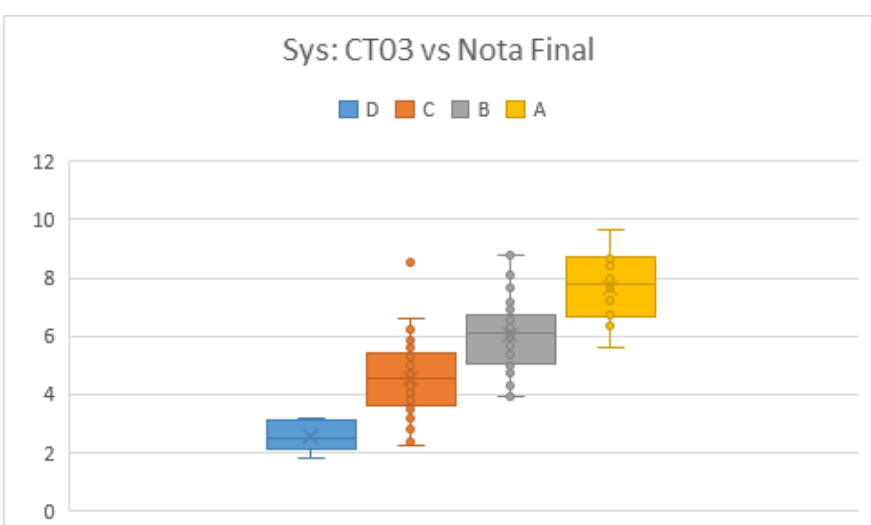

Fig. 2 Evaluación de la competencia CT3 en la asignatura Señales y Sistemas

(cc) EY-NC-No 2016, Universitat Politècnica de València

Congreso In-Red (2016) 
Este efecto es lógico y es debido a que se están utilizando los mismos problemas (aunque con preguntas específicas en ciertos apartados) para valorar una parte de la nota final (en este caso el tercer parcial y final de la asignatura) y la propia competencia transversal.

Es de destacar que, aun existiendo una clara correlación de los datos, no todos los alumnos con una B en la competencia aprueban la asignatura, ya que en realidad no se está evaluando exactamente lo mismo. Incluso hay casos de alumnos con una $\mathrm{C}$ en la competencia que aprueban e incluso en algún caso sacan buena nota en la asignatura. Pero claramente son casos aislados.

En la Tabla 4 y Figura 3 se muestran los resultados en la asignatura Matemáticas II.

Tabla 4. Datos referentes a la asignatura Matemáticas II

\begin{tabular}{|c|c|c|c|c|c|c|}
\hline Matemáticas II & Media & Desviación típica & Max caja & Min caja & Max & Min \\
\hline D & 1,90 & 0,00 & 1,90 & 1,90 & 1,9 & 1,9 \\
\hline C & 6,10 & 0,87 & 6,97 & 5,23 & 7,6 & 5,1 \\
\hline B & 6,92 & 0,61 & 7,52 & 6,31 & 7,9 & 5,9 \\
\hline A & 8,76 & 1,13 & 9,89 & 7,63 & 9,6 & 8 \\
\hline
\end{tabular}

Matematicas II: CT03 vs Nota Final

$\square \mathrm{D} \square \mathrm{C} \square \mathrm{B} \square \mathrm{A}$

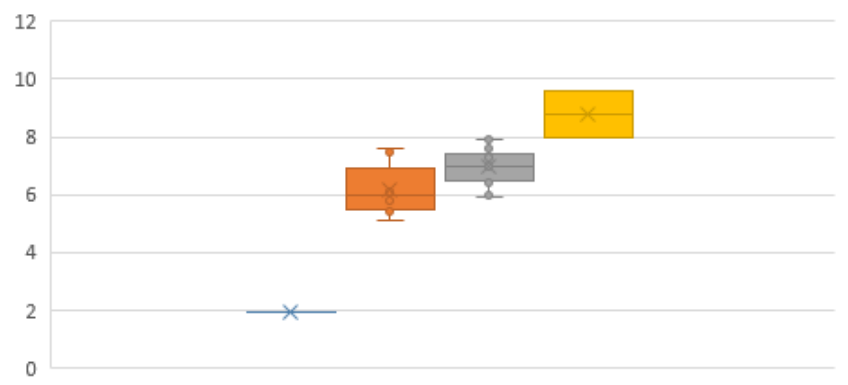

Fig. 3 Evaluación de la competencia en la asignatura Matemáticas II

Existe, al igual que en los casos anteriores, una alta correlación entre la nota final y la nota de la competencia. Esto es debido a la aplicación directa del resultado obtenido en los problemas evaluados, que corresponde a un $60 \%$ de la nota final de la asignatura, para obtener la calificación de la competencia.

En la Tabla 5 y Figura 4 se muestran los resultados en la asignatura Reactores químicos.

El análisis de los resultados muestra que hay una correlación positiva entre el nivel de desarrollo de la competencia y la nota final del alumno en la asignatura. Los alumnos con nota final inferior a 6 obtienen calificaciones de D (no alcanzado) y C (en desarrollo) para la competencia. Los alumnos con nota final entre 6 y 7 obtienen una calificación de B (adecuado) y sólo los alumnos que presentan más de un 7 en la nota final obtienen una calificación de A (excelente). Esta correlación positiva era esperable ya que 2 créditos de la asignatura corresponden a las prácticas que consisten en la resolución de problemas. Se 
observan un valor atípico de nota final de 5.2 y calificación en el desarrollo de la competencia de adecuado.

Tabla 5. Datos referentes a la asignatura Reactores Químicos

\begin{tabular}{|c|c|c|c|c|c|c|}
\hline Reactores Químicos & Media & Desviación típica & Max caja & Min caja & Max & Min \\
\hline D & 4,90 & 0,70 & 5,60 & 4,19 & 5,5 & 3,4 \\
\hline C & 5,17 & 0,60 & 5,77 & 4,58 & 6,1 & 4 \\
\hline B & 6,30 & 0,40 & 6,70 & 5,90 & 7 & 5,2 \\
\hline A & 7,63 & 0,39 & 8,02 & 7,24 & 8,2 & 7,1 \\
\hline
\end{tabular}

Reactores: CT03 vs Nota Final

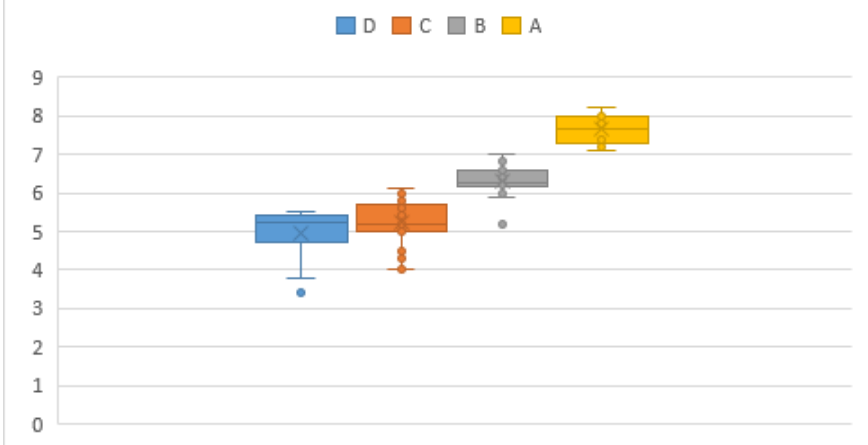

Fig. 4 Evaluación de la competencia en la asignatura Reactores químicos

Destacar que en este caso la correlación no es tan evidente, sobre todo en las valoraciones de la D y la C, aunque se ve más clara en la B y la A.

\section{Conclusiones}

Tras trabajar en diversas asignaturas de grado de diversas titulaciones de la UPV la competencia transversal “Análisis y resolución de problemas”, podemos concluir que esta nota obtenida por los alumnos en ella tiene un alto índice de correlación con la nota final de la asignatura. Lo que resulta coherente si se considera que para la calificación de la competencia se han tenido en cuenta algunas de las notas obtenidas en los actos de evaluación de estas asignaturas.

Por ello, para trabajar esta competencia, creemos interesante señalar las siguientes recomendaciones:

Dar a conocer la rúbrica con la que vamos a evaluar la competencia a principio de curso, para que los alumnos la tengan disponible desde el primer día de clase y puedan empezar a trabajar desde las primeras sesiones de problemas con un carácter formativo.

Utilizar esta rúbrica a la hora de valorar las preguntas específicas de cada problema, informando a los alumnos de sus progresos desde las primeras sesiones de problemas.

(cc) EY-Nc-ND 2016, Universitat Politècnica de València

Congreso In-Red (2016) 
Calificar la competencia considerando cada caso, sin fórmulas proporcionales a la nota final de la asignatura, fijándonos en los ítems correspondientes a cada resultado de aprendizaje y siempre con una visión de conjunto de la trayectoria del alumno.

En trabajos futuros se pretende realizar una comparación de los materiales desarrollados en cada asignatura para el diseño y propuesta de actividades de enseñanza-aprendizaje de esta competencia transversal. Además, se pretende valorar el diseño de los instrumentos de evaluación para permitir la obtención de evidencias concretas, integradas con las competencias específicas de cada asignatura, en función de la ubicación de cada materia en las correspondiente titulaciones de la UPV.

\section{Referencias}

GIORDANO, D., MAIORANA, F. (2014), "Use of Cutting Edge Educational Tools for an Initial Programming Course” en IEEE Global Engineering Education Conference (EDUCON). Disponible en <http://toc.proceedings.com/22383webtoc.pdf> [Consulta: 1 de abril de 2016]

GLEASON, B. L., GAEBELEIN, C. J., GRICE, G. R., CRANNAGE, A. J., WECK, M. A., HURD, P., WALTER, B., DUNCAN, W. (20013) “Assessment of Students' Critical-Thinking and ProblemSolving Abilities Across a 6-Year Doctor of Pharmacy Program”. American journal of $\begin{array}{lllll}\text { pharmaceutical } & \text { education, } & \text { Vol. } & 77 & \text { (8), }\end{array}$ http://www.ncbi.nlm.nih.gov/pmc/articles/PMC3806950/> [Consulta: 1 de abril de 2016]

GONZÁLEZ, J.,WAGENAAR, R. (2003). Tuning Educational Structures in Europe. Informe Final. Bilbao: Universidad de Deusto

HUNG, W. (2013) “Team-based complex problem solving: a collective cognition perspective” Educational Technology Research and Development, Vol. 61 (3) pp 365-384. < http://link.springer.com/article/10.1007/s11423-013-9296-3/fulltext.html> [Consulta: 1 de abril de 2016]

LLORET, J., JIMÉNEZ, J. M., BORONAT, F., TOMÁS, J., DÍAZ, J.R., Utilización de diversas metodologías didácticas para desarrollar las habilidades de los estudiantes de Ingeniería Técnica de Telecomunicaciones, Congreso Internacional de Docencia Universitaria e Innovación (CIDUI 2006), Barcelona, España, Barcelona 5 - 7 de Julio 2006.

OECD. PISA 2012 Field Trial Problem Solving Framework. 30 September 2010 <http://www.oecd.org/dataoecd/8/42/46962005.pdf>.[Consulta: 1 de abril de 2016]

Real Decreto 1125/2003, de 5 de septiembre, por el que se establece el sistema europeo de créditos y el sistema de calificaciones en las titulaciones universitarias de carácter oficial y validez en todo el territorio nacional. BOE, 18 de Septiembre de 2003, núm. 224

SANABRIA-CODESAL, E., BOSCH, I., VINCENT-VELA, M.-C., LLORET, J., ÁLVAREZBLANCO, S., ROMERO PÉREZ, L., Análisis de las Dimensiones Competenciales Incluidas en Diferentes Asignaturas en Ingenierías, Jornadas de Innovación Educativa y Docencia en Red (In-Red 2014), Valencia (España), 15-16 de Julio 2014

SÁNCHEZ-BARBA MERLO, L. F., GARCÉS OSADO, A., HONRADO, M. (2013), "Development and assessment of problem solving skill to promote learning in chemistry" en 5th International Conference on Education and New Learning Technologies (EDULEARN). Disponible en <https://library.iated.org/view/SANCHEZBARBAMERLO2013DEV.> [Consulta: 1 de abril de 2016]

(cc) EY-NC-ND 2016, Universitat Politècnica de València 
SENDRA, S., CÁNOVAS, A., GARCÍA, M., LLORET, J., Método de evaluación cooperativa en clases prácticas de redes de ordenadores, EAA-Jornadas de Innovación, Julio 8-10, 2009. Valencia, España.

UNIVERSITAT POLITÈCNICA DE VALÈNCIA. Proyecto institucional Incorporación de las competencias transversales en el currículo de los egresados de la UPV. $<$ http://www.upv.es/entidades/ICE/info/U0702452.pdf > [Consulta: 1 de abril de 2016]

UNIVERSITAT POLITÈCNICA DE VALÈNCIA. INSTITUTO DE CIENCIAS DE LA EDUCACIÓN. Actividades formativas para trabajar las competencias transversales. $<$ https://poliformat.upv.es/access/content/group/ESP_0_2254/04.\%20Material\%20complementario/A ctividades\%20formativas\%20recomendadas\%20CT-UPV.pdf>. [Consulta: 8 de abril de 2016]

VICENT L., ÀVILA X., RIERA, J., ANGUERA J., BADIA D., MONTERO J. (2006), "Appropriateness of e-learning resources for the development of transversal skills in the new European Higher Education Area” en Frontiers in Education Conference < http://ieeexplore.ieee.org/stamp/stamp.jsp?tp=\&arnumber=4117111> [Consulta: 1 de abril de 2016] 